\title{
Topographic Diagnosis of Papillary Craniopharyngiomas: The Need for an Accurate MRI-Surgical Correlation
}

W e have read with great interest the original article by Lee et al, " "Pretreatment Diagnosis of Suprasellar Papillary Craniopharyngioma and Germ Cell Tumors of Adult Patients," recently published in the American Journal of Neuroradiology, which analyzes the MR imaging features of a series of 18 papillary craniopharyngiomas (PCPs), to achieve an accurate differential diagnosis between these lesions and the group of germ cell tumors involving the infundibulotuberal area. First and foremost, we would like to congratulate the authors for their thorough survey on such a challenging topographic variant of craniopharyngioma (CP), a topic rarely addressed in neuroradiologic research. With the present communication, we wish to highlight some important pathologic and topographic concepts regarding PCPs that should be taken into account to avoid erroneous interpretations of MR imaging studies.

In their article, Lee et $\mathrm{al}^{1}$ defined PCPs as suprasellar lesions, presumably originating from the pars tuberalis, the thin layer of glandular tissue enveloping the pituitary stalk and the outer surface of the infundibulum. Consequently, these tumors are considered to expand preferentially outside the third ventricle, into the subarachnoid spaces that form the chiasmatic or suprasellar cistern. This concept assumes that PCPs represent extraventricular tumors that may involve, secondarily, the third ventricle, in contrast to germ cell tumors (GCTs), which would have a primary location at the level of the third ventricle floor and infundibular recess. In disagreement with these statements, the authors observed the highest rate of third ventricle occupation among PCPs ( $83 \%$ of cases). Contrary to GCTs, most PCPs showed a smooth spheric shape without any transinfundibular infiltration of the pituitary stalk. Most interesting, the MR imaging scans of the 2 PCP cases displayed in the article (Figs 1 and 2) evidenced, respectively, a major occupation of the third ventricle floor and of the third ventricle cavity by the tumor, despite both lesions being classified within the "suprasellar" topography. In particular, the second PCP displayed in Fig $2 A$ seems to be a truly intraventricular tumor, developing above an intact third ventricle floor, which can be easily identified beneath the lower pole of the tumor.

http://dx.doi.org/10.3174/ajnr.A4441
Both the suprasellar cistern and the pituitary stalk are free of tumor in this latter case. In addition, the hyperintense linear signal corresponding to the median eminence can be observed beneath the tumor. The top of the lesion blocks the foramen of Monro and protrudes through the columns of the fornix.

No details about the type of surgical procedure or the surgical findings observed in each case are provided in the article, which precludes a confirmation of the true location and extension of the PCP displayed in Fig 2. Yet, we argue for a redefinition of this tumor as a strictly intraventricular PCP. ${ }^{2-5}$ In the case of lesions grossly replacing the infundibulotuberal area of the third ventricle floor, such as the tumor displayed in Fig 1, we advocate the use of the terms "not strictly intraventricular" or "infundibulotuberal," which stress their original development within the neural tissue of the infundibulum and/or tuber cinereum..$^{2-5}$ This latter subgroup, exceedingly frequent among adult PCPs, is characterized by its bidirectional growth toward the third ventricle cavity and the suprasellar cistern, with the center of the mass expanding at the level of the hypothalamus proper.

The classic, prevailing notion regarding the topography of craniopharyngiomas considers a general extra-axial or suprasellar location for these lesions. Such a misleading assumption is based on the presumed embryologic origin of CPs from epithelial remnants of the Rathke pouch, a structure derived from the primitive stomodeum. However, because of the original description of CPs by Jakob Erdheim in 1904, many pathologic reports of nonoperated CPs found in postmortem examinations evidenced tumors hidden within the third ventricle, which had developed at the infundibulotuberal area of the basal hypothalamus and respected the integrity of the pituitary gland and pituitary stalk. ${ }^{4}$ Many of these lesions were diagnosed in adults and corresponded to the squamous-papillary histologic variant. The spheric morphology displayed by PCPs contrasts markedly with the multilobulated shape shown by adamantinomatous CPs. This difference may depend not only on the intrinsic characteristics of each histologic variant but rather on the original position that epithelial remnants occupy along the pituitary axis. ${ }^{3-5}$ A subpial inclusion of epithelial cell remnants of the craniopharyngeal duct into the third ventricle floor at the time the Rathke pouch contacted the 
diencephalic vesicle would account for the development of both strictly intraventricular and infundibulotuberal $\mathrm{CPs}^{3,4}$

Because of the usual tight attachment occurring between the hypothalamus and the $\mathrm{CP}$ when the tumor develops primarily at the level of the third ventricle floor, an accurate preoperative MR imaging identification of the position and integrity of the infundibulotuberal area is of the utmost importance. ${ }^{3}$ However, a clear demarcation between the strictly intraventricular and the infundibulotuberal categories can hardly be made in most cases, even with the use of high-resolution MR imaging, because the integrity of the third ventricle floor cannot usually be defined preoperatively. ${ }^{2,5}$ The relative position and morphology of the mammillary bodies with respect to the tumor can be helpful to ascertain the anatomic origin of the mass along the pituitaryhypothalamic axis. ${ }^{5}$ Nevertheless, CP topography can be reliably confirmed only at the end of the surgical procedure, once the lesion has been totally removed.

In 2004, our group proposed a reclassification of the CP topography according to the type of third ventricle floor involvement by the lesion. Among the 33 verified PCPs analyzed in the medical literature up to that time, two-thirds of lesions corresponded to strictly third ventricle tumors, and one-third, to infundibulotuberal lesions. ${ }^{2}$ In a thorough subsequent review of infundibulotuberal CPs described in surgical series and postmortem examinations, including almost 1500 tumors, it was shown that approximately $30 \%$ of these lesions corresponded to PCPs. ${ }^{4}$ Finally, in a recent analysis of MR imaging signs allowing an accurate preoperative identification of $\mathrm{CP}$-third ventricle relationships, only 3 of 24 verified PCPs were extraventricular lesions developing exclusively within the suprasellar cistern, below an intact third ventricle floor. ${ }^{5}$ Most of the lesions corresponded to either strictly intraventricular or primarily infundibulotuberal CPs.

To conclude, we think that the general term "suprasellar" used by most authors to define the location of CPs showing both ex- traventricular and intraventricular extensions is too imprecise, and it should be avoided when categorizing the topography of CPs involving the third ventricle. We favor instead the term "infundibulotuberal" to designate the important group of CPs developing in the proper neural tissue of the third ventricle floor. A significant percentage of PCPs belongs to this category.

\section{REFERENCES}

1. Lee HJ, Wu CC, Wu HM, et al. Pretreatment diagnosis of suprasellar papillary craniopharyngioma and germ cell tumors of adult patients. AJNR Am J Neuroradiol 2015;36:508-17

2. Pascual JM, Gonzalez-Llanos F, Barrios L, et al. Intraventricular craniopharyngiomas: topographical classification and surgical approach selection based on an extensive overview. Acta Neurochir (Wien) 2004;146:785-802

3. Pascual JM, Carrasco R, Prieto R, et al. Craniopharyngioma classification. J Neurosurg 2008;109:1180-82; author reply 1182-83

4. Pascual JM, Prieto R, Carrasco R. Infundibulo-tuberal or not strictly intraventricular craniopharyngioma: evidence for a major topographical category. Acta Neurochir (Wien) 2011;153:2403-25; discussion 2426

5. Pascual JM, Prieto R, Carrasco R, et al. Displacement of mammillary bodies by craniopharyngiomas involving the third ventricle: surgical-MRI correlation and use in topographical diagnosis. J Neurosurg 2013;119:381-405

J.M. Pascual

Department of Neurosurgery

La Princesa University Hospital Madrid, Spain

R. Prieto

Department of Neurosurgery Puerta de Hierro University Hospital Madrid, Spain I. Castro-Dufourny

Department of Endocrinology Hospital del Sureste Madrid, Spain

R. Carrasco

Department of Neurosurgery Ramón y Cajal University Hospital Madrid, Spain 